01,07

\title{
Прогнозирование динамического предела текучести металлов с помощью двух структурно-временных параметров
}

\author{
(C) Н.С. Селютина ${ }^{1,2}$, Ю.В. Петров ${ }^{1,2}$ \\ ${ }^{1}$ Институт проблем машиноведения РАН, \\ Санкт-Петербург, Россия \\ ${ }^{2}$ Санкт-Петербургский государственный университет, \\ Санкт-Петербург, Россия \\ E-mail: nina.selutina@gmail.com
}

(Поступила в Редакцию 26 июля 2017 г.)

На основе критерия инкубационного времени текучести и эмпирических моделей Джонсона-Кука и Cowper-Symonds исследуется поведение предела текучести стали и ряда алюминиевых сплавов в широком диапазоне скоростей деформации. В работе выведены выражения для параметров эмпирических моделей через характеристики критерия инкубационного времени текучести и получено удовлетворительное соответствие при их сравнении с экспериментальными данными. Показано, что параметры эмпирических моделей могут зависеть от некоторой скорости деформации. Независимость характеристик критерия инкубационного времени текучести от истории нагружения и их связь со структурно-временными особенностями процесса пластического деформирования дает преимущество подхода, базирующегося на понятии инкубационного времени, относительно эмпирических моделей, а также эффективную и удобную формулу для определения предела текучести в более широком диапазоне скоростей деформаций.

Исследование выполнено за счет гранта Российского научного фонда (проект 17-71-10061).

DOI: 10.21883/FTT.2018.02.45374.239

\section{1. Введение}

Развитие пластической деформации в металлах при высокоскоростном нагружении напрямую зависит от уровня приложенной нагрузки. Наблюдаемые на практике динамические эффекты пластичности сложно объяснить только в рамках классической теории пластичности (условия минимального критического напряжения, такие как критерии Мизеса и Треска). В частности, это проявляется в нестабильном поведении предела текучести в зависимости от скорости деформации. На уровне дефектной структуры металла причиной может являться влияние временного фактора на механизм образования пластической деформации, который существенно отличается в условиях динамики от условий статики [1].

На данный момент отсутствует феноменологический подход, способный прогнозировать динамические эффекты пластического деформирования и одновременно обладающий физически обоснованными, не зависимыми от скорости деформации параметрами материала. Прогнозирование динамических эффектов при пластическом деформировании металлов обычно проводится с помощью методов численного моделирования. В частности, широко применяются следующие эмпирические модели: Cowper-Symonds [2], ДжонсонаКука [3,4], Зерилли-Амстронга [5], Стейнберг-КочранГюнан-Ланда [6], Престон-Тонкс-Валласа [7]. Новые эксперементальные данные по динамическому пределу текучести показали, что существующие модели не всегда описывают его поведение на более высоких скоростях деформации. Примером является неприменимость формулы для предела текучести в рамках классической модели Джонсона-Кука [3,4] на высоких скоростях деформации $10^{3} \mathrm{~s}^{-1}$ и формулировка ее модификации [8], способной описывать поведение динамического предела текучести в широком диапазоне скоростей деформации. Таким образом, формулировка модифицированных моделей Huh-Kang [9], Tuazon [10], Couque [8], Eyring [11] с дополнительными эмпирическими параметрами позволяет численно решить проблему описания наблюдаемых динамических эффектов пластичности. При этом каждый из новых параметров модифицированных моделей обычно трудно связать с физической природой динамики пластического деформирования, так как вводимые параметры только условно описывают нелинейность процесса и являются эмпирическими.

В работе [12] были получены аналитические выражения для параметров модифицированной модели Джонсона-Кука через характеристики критерия инкубационного времени текучести [13-16]. Построенные соотношения выявили зависимость эмпирических параметров от скорости деформации, что является существенным недостатком численных моделей.

Разрабатываемый структурно-временной подход на основе действия инкубационного времени [13-16] может быть использован в качестве альтернативного подхода для описания временных эффектов при высокоскоростном деформировании металлов. Критерий инкубационного времени текучести позволяет прогнозировать поведение предела текучести в более широком диапазоне скоростей деформации в сравнении с классической формулой Джонсона-Кука и ее модификациями [12]. Это возможно за счет внедрения в расчеты постоянного 
параметра инкубационного времени, учитывающего процессы релаксации напряжений при переходе на стадию пластического деформирования как временные и связанные с движением дефектов. Как было установлено ранее $[1,17,18]$, параметр инкубационного времени текучести не зависит от механизма пластичности, а только от дефектной структуры материала.

В представленной работе дана физическая интерпретация параметров модели Cowper-Symonds [2] с помощью характеристик критерия инкубационного времени текучести. В качестве эмпирической модели, модель Cowper-Symonds является наиболее предпочтительной относительно модифицированной модели ДжонсонаКука [8] при определении предела текучести в начальный момент пластического деформирования, так как ее параметры не зависят от скорости деформации. Сравнение структурно-временного подхода с известными численными моделями позволяет установить диапазон допустимых значений параметров эмпирических моделей.

\section{2. Критерий инкубационного времени}

Структурно-временной подход для описания процессов текучести позволяет рассчитывать предел текучести в начальный момент пластической деформации при фиксированной скорости деформации [13-16]. Изначально структурно-временной подход [19] был сформулирован для описания процессов разрушения [20] и являлся эффективным инструментом для описания временных эффектов роста микротрещин [21,22]. Предложенный макроскопический критерий текучести [13-16] для случая одноосного напряженного состояния, именуемый в дальнейшем критерием инкубационного времени, определяется неравенством

$$
\frac{1}{\tau} \int_{t-\tau}^{t}\left(\frac{\Sigma(s)}{\sigma_{y}}\right)^{\alpha} d s \leq 1 .
$$

Здесь $\Sigma(t)$ - функция напряжений от времени, $\tau-$ инкубационное время, $\sigma_{y}-$ статический предел текучести, $\alpha-$ коэффициент чувствительности материала к амплитуде нагрузки. Заметим, что начало макроскопической текучести $t_{*}$ определяется из условия равенства (1). Вводимый временной параметр $\tau$, независящий от особенностей процесса деформации и геометрии образца, позволяет прогнозировать поведение предела текучести материала в рамках статических и динамических нагрузок $[15,16]$. Предполагается, что параметр инкубационного времени может принимать различные значения в зависимости от начальной дефектной структуры материала. Другими словами, два образца, сделанные из одного и того же материала в результате двух различных технологических процессов, с разной структурой до начала испытаний на пластическое деформирование будут рассматриваться как образцы, сделанные из двух разных материалов. Таким образом, два независимых параметра критерия (1) $\tau$ и $\alpha$ характеризуют скоростную (временну́ю) и амплитудную чувствительности материала соответственно. Для большинства металлов $\alpha=1$ дает хорошее соответствие с экспериментальными данными. В данной работе также будет рассмотрен пример высокоскоростного пластического деформирования стали с $\alpha \neq 1$ [23]. Отметим, что для случаев сложного напряженного состояния критерий (1) может быть перезаписан в форме инвариантов [14].

Получим скоростную зависимость предела текучести от скорости деформации. Рассмотрим линейный закон упругого деформирования, $\Sigma(t)=E \dot{\varepsilon} t H(t)(E-$ модуль Юнга, $\dot{\varepsilon}$ - постоянная скорость деформации при нагрузке). Записывая левую часть (1) при условии наступления текучести при времени $t_{*}$, можно выразить динамический предел текучести $\Sigma_{d}=\Sigma\left(t_{*}\right)$ в зависимости от скорости деформации материала

$$
\begin{aligned}
& \Sigma_{d}(\dot{\varepsilon})= \\
& = \begin{cases}{\left[(\alpha+1)\left(\sigma_{y}\right)^{\alpha} E \dot{\varepsilon} \tau\right]^{1 /(\alpha+1)},} & \dot{\varepsilon} \geq \frac{(\alpha+1)^{1 / \alpha} \sigma_{y}}{E \tau}\left(t_{*} \leq \tau\right) ; \\
\sigma_{y}+\left(1-\frac{1}{(\alpha+1)^{1 / \alpha}}\right) E \dot{\varepsilon} \tau, & \dot{\varepsilon}<\frac{(\alpha+1)^{1 / \alpha} \sigma_{y}}{E \tau}\left(t_{*}>\tau\right) .\end{cases}
\end{aligned}
$$

Полученная расчетная формула состоит из двух частей: линейная (статические воздействия) и нелинейная (динамические воздействия), разделяемая условной точкой перехода по скорости деформации $(\alpha+1)^{1 / \alpha} \sigma_{y} /(E \tau)$. Нижнее выражение в правой части уравнения (2) описывает случай деформирования с линейным увеличением динамического предела текучести со скоростью деформации (время процесса сравнимо или выше инкубационного времени $\tau$ ). Верхнее выражение соответствует противоположному случаю, когда время нагрузки меньше, чем $\tau$. Рассматривая экспериментальные точки предела текучести в зависимости от скорости деформации, полученные по формуле (2), можно оценить методом наименьших квадратов инкубационное время. Таким образом, набор независимых параметров $\left(\sigma_{y}, \tau, \alpha\right)$ описывает поведение предела текучести материала в широком диапазоне скоростей деформации вне зависимости от модели пластичности и способа воздействия.

\section{3. Численные модели для оценки динамического предела текучести}

В инженерной практике, связанной с вопросами пластического высокоскоростного деформирования металлов, широко используют ряд эмпирических моделей. Рассмотрим и проанализируем часть из них для предсказания динамического предела текучести в начальный момент пластического деформирования без учета дальнейшего упрочнения и термического влияния.

Оценка предела текучести по модели CowperSymonds [12] осуществляется в следующей форме на 
основе эмпирических параметров $B, q$ :

$$
\Sigma_{C S}(\dot{\varepsilon})=\sigma_{y}\left(1+\left(\frac{\dot{\varepsilon}}{B}\right)^{1 / q}\right) .
$$

Проводя аналогии между критерием инкубационного времени текучести и моделью Cowper-Symonds (7), построим соотношения для параметров $B, q$

для случая $t_{*}>\tau$ :

$$
q=\alpha+1, B(\dot{\varepsilon})=\left[\left(1-\frac{1}{(\alpha+1)^{1 / \alpha}}\right) \frac{E \tau}{\sigma_{y}}\right]^{-\alpha-1} \dot{\varepsilon}^{-\alpha}
$$

и для случая $t_{*} \leq \tau$ :

$$
q=\alpha+1, \quad B=\frac{\sigma_{y}}{E \tau(\alpha+1)}
$$

Таким образом, один из параметров модели CowperSymonds выражается через параметр амплитудной чувствительности материала, другой - через оба независимых параметра $\tau, \alpha$. При этом в формуле (4) параметр $B$ зависит от скорости деформации.

Классическая модель Джонсона-Кука $[3,4]$ и ее модификация [8] задаются формулами

$$
\begin{gathered}
\Sigma_{J K}(\dot{\varepsilon})=A_{J K}\left(1+C \ln \left(\frac{\dot{\varepsilon}}{\dot{\varepsilon}_{0}}\right)\right), \\
\Sigma_{M J K}(\dot{\varepsilon})=A_{J K}\left(1+C \ln \left(\frac{\dot{\varepsilon}}{\dot{\varepsilon}_{0}}\right)+D\left(\frac{\dot{\varepsilon}}{\dot{\varepsilon}_{1}}\right)^{k}\right),
\end{gathered}
$$

где $A_{J K}, C, n, m-$ постоянные параметры классической модели Джонсона-Кука (6); $\dot{\varepsilon}-$ пластическая скорость деформации; $\dot{\varepsilon}_{1}=1000 \mathrm{~s}^{-1}$ по [8]; $T-$ температура; $\dot{\varepsilon}_{0}-$ пластическая скорость деформации $\left(\dot{\varepsilon}_{0}=1 \mathrm{~s}^{-1}\right.$ в $[3,4,24]$ и $\dot{\varepsilon}_{0}=0.001 \mathrm{~s}^{-1}$ в [25]); $D, k$ - константы модифицированной модели (7).

Как было показано в [12], выражения для параметров модели Джонсона-Кука (6), (7) записываются через характеристики критерия инкубационного времени (1) в следующей форме:

$$
\begin{gathered}
A_{J K}=\sigma_{y}, C\left(\dot{\varepsilon}_{0}\right)=\left(1-\frac{1}{(\alpha+1)^{1 / \alpha}}\right) \frac{E \dot{\varepsilon} \tau}{\sigma_{y}}\left[\ln \left(\frac{\dot{\varepsilon}}{\dot{\varepsilon}_{0}}\right)\right]^{-1} ; \\
k=\frac{1}{\alpha+1}, D\left(\dot{\varepsilon}_{1}\right)=\left((\alpha+1) \frac{E \dot{\varepsilon}_{1} \tau}{\sigma_{y}}\right)^{1 /(\alpha+1)}
\end{gathered}
$$

Эмпирические параметры формулы Джонсона-Кука $D$ и $C$ являются зависимыми от фиксированной скорости деформации, и параметр $k$ имеет обратно пропорциональную зависимость от параметра амплитудной чувствительности материала $\alpha$. При этом поведение предела текучести на высоких скоростях деформации лучше описывает модифицированная модель Джонсона-Кука. Таким образом, два параметра характеризуют поведение динамического предела текучести как в модели Джонсона-Кука, так и в модели Cowper-Symonds, и один из них зависит от скорости деформации.

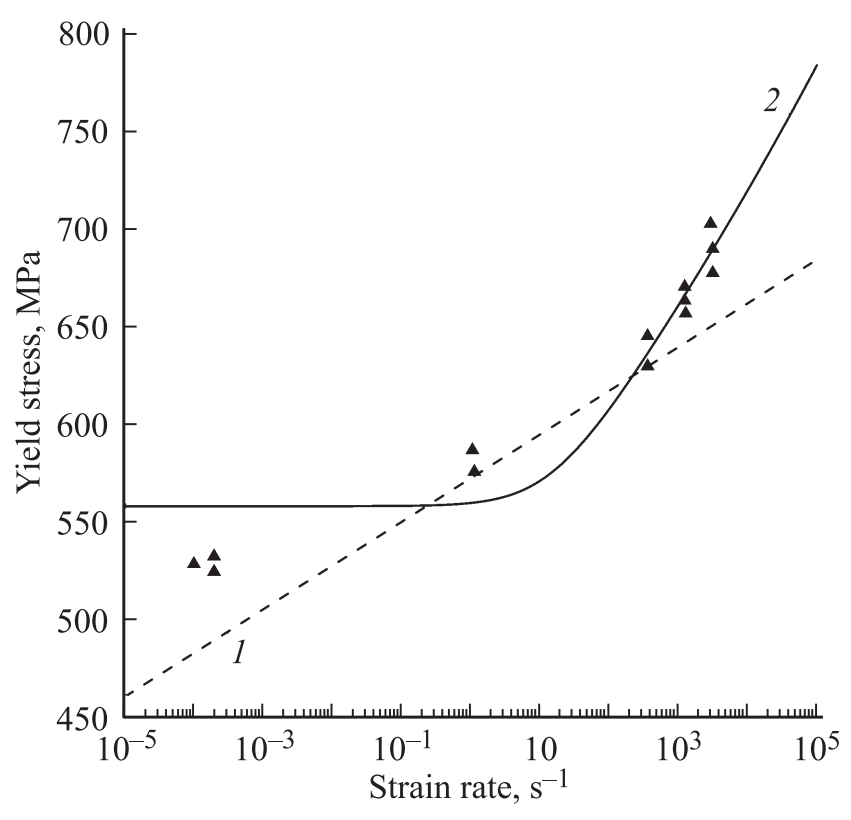

Рис. 1. Зависимость предела текучести от скорости деформации для стали ASTM A36 (экспериментальные значения [24] обозначены треугольниками) по классической модели Джонсона-Кука (6) (кривая 1) и критерию (1) (кривая 2).

На примере стали [24] $\left(\sigma_{y}=558 \mathrm{MPa}, E=210 \mathrm{GPa}\right)$ показано, что „линейный ${ }^{\circ}$ рост предела текучести со скоростью деформации по классической модели Джонсона-Кука (6) $\left(A_{J K}=530 \mathrm{MPa}, C=0.0017\right.$, $\dot{\varepsilon}_{0}=1 \mathrm{~s}^{-1}$ ) формально дает хорошее соответствие с экспериментальными данными (рис. 1, кривая 1). Применение критерия (1) $(\alpha=26, \tau=9.58 \mu \mathrm{s})$ позволяет предсказывать нестабильное поведение предела текучести в широком диапазоне скоростей деформации (рис. 1, кривая 2). Тогда по (8) параметр $C$ при скорости деформации $10 \mathrm{~s}^{-1}$ равен 0.00186 и совпадает численно с полученным в работе [24].

Рассмотрим экспериментальные данные для алюминиевого сплава 7075-Т6 [25] и никеля [26]. На рис. 2 и 3 показаны зависимости предела текучести в широком диапазоне скоростей деформации, построенные по моделям Джонсона-Кука (6), (7) (рис. 2: $A=473 \mathrm{MPa}$, $C=0.033$; рис. $3: A=200 \mathrm{MPa}, C=0.01, D=0.25$, $k=0.5$ ) и модели (1) (рис. $2: \sigma_{y}=473 \mathrm{MPa}, \tau=6.5 \mu \mathrm{s}$, $\alpha=1$; рис. 3: $\left.\sigma_{y}=240 \mathrm{MPa}, \tau=0.5 \mu \mathrm{s}, \alpha=1\right)$. Модель (1), (7) дает хорошее соответствие с экспериментальными данными как в статике, так и в динамике (рис. 3). Как показано на рис. 3, классическая модель Джонсона-Кука (6) дает удовлетворительную оценку предела текучести только до скорости деформации порядка $10^{3} \mathrm{~s}^{-1}$. Характеристики модели Джонсона-Кука были вычислены через параметры критерия (3): $C=0.036, D=1.4$ (при $\dot{\varepsilon}=1000 \mathrm{~s}^{-1}$ ), $k=0.5[25] ; C=0.032$ (при $\left.\dot{\varepsilon}=1000 \mathrm{~s}^{-1}\right), \quad D=0.9$ (при $\dot{\varepsilon}=1000 \mathrm{~s}^{-1}$ ), $k=0.5[26]$.

На рис. 4 представлены скоростные зависимости предела текучести для алюминиевого сплава 


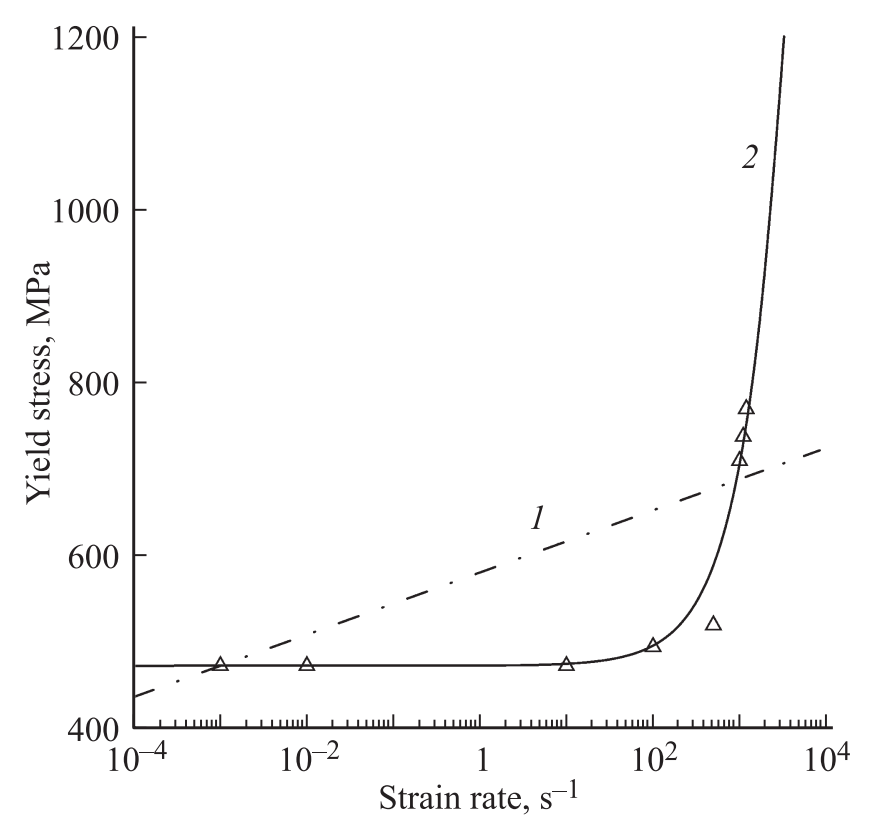

Рис. 2. Зависимость предела текучести от скорости деформации для алюминиевого сплава 7075-Т6 (экспериментальные значения [25] обозначны треугольниками) по классической модели Джонсона-Кука (6) (кривая 1) и критерию (1) (кривая 2).

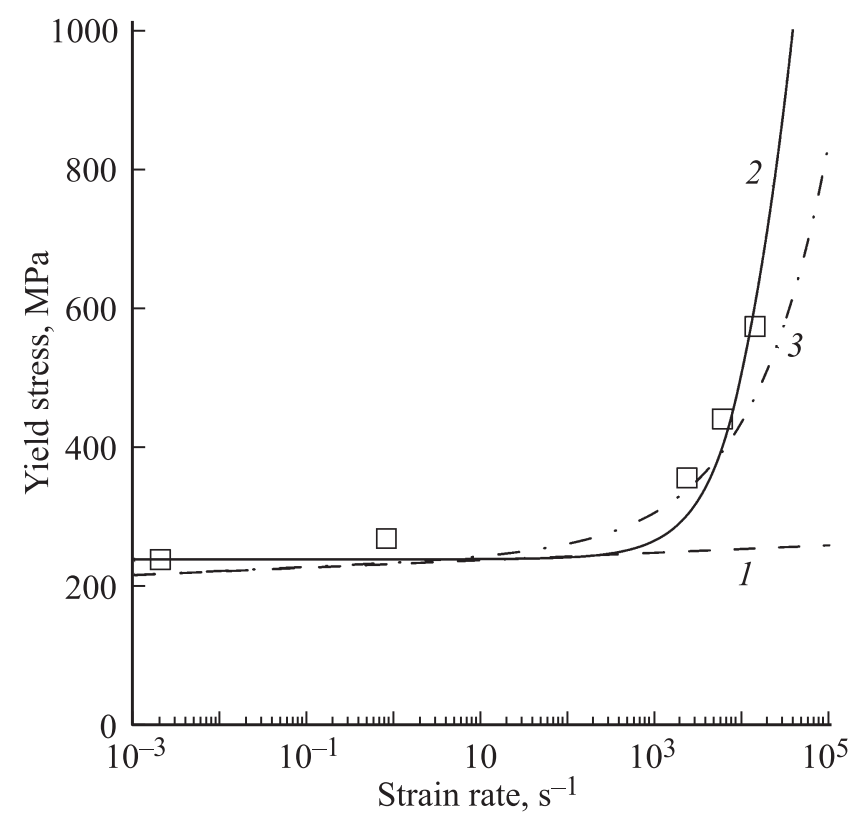

Рис. 3. Зависимость предела текучести от скорости деформации для никеля (экспериментальные значения [26] обозначны квадратами) по классической модели Джонсона-Кука (6) (кривая 1) и критерию (1) (кривая 2), по модифицированной модели Джонсона-Кука (7) (кривая 3).

7449-T7651 [27] $\left(\sigma_{y}=519 \mathrm{MPa}, E=70 \mathrm{GPa}\right)$, построенные по модели Cowper-Symonds $\left(B=15007 \mathrm{~s}^{-1}\right.$, $q=0.95)$, которые могут лучше совпадать с прогнозированием по критерию инкубационного времени текучести $(\alpha=1, \tau=0.94 \mu \mathrm{s})$, как показано на рис. 2 на примере алюминиевого сплава.
Рассмотрим экспериментальные данные для стали [28] $\left(\sigma_{y}=560 \mathrm{MPa}, E=210 \mathrm{GPa}\right)$ и проведем сравнение расчетных схем динамического предела текучести по критерию инкубационного времени (2) $(\alpha=1, \tau=1 \mu \mathrm{s})$, модели Cowper-Symonds (3) и классической моде-

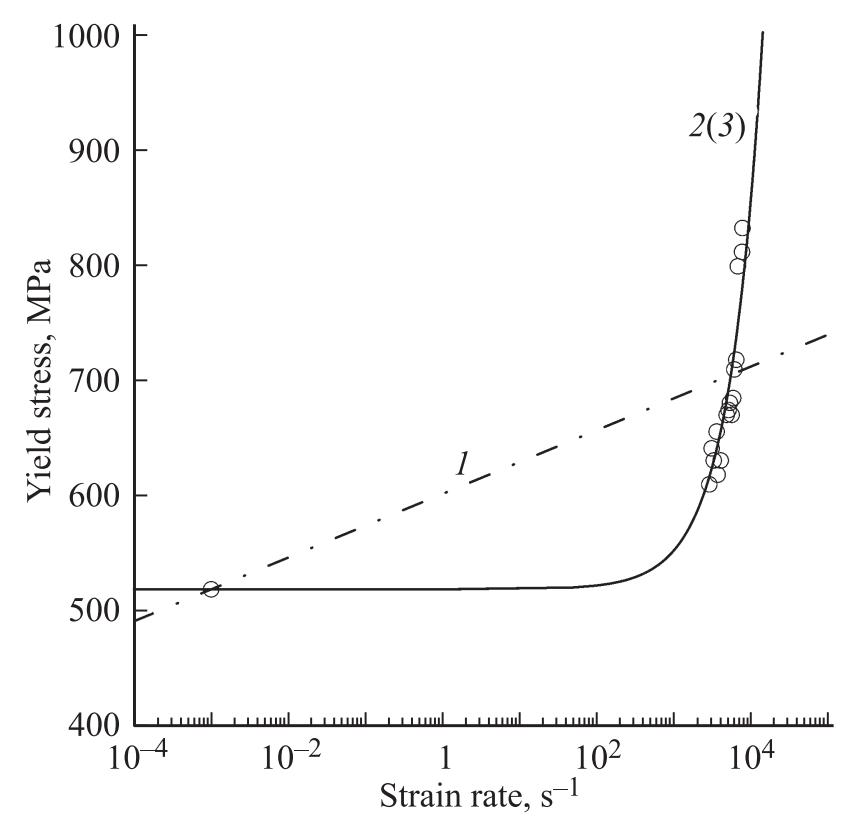

Рис. 4. Зависимость предела текучести от скорости деформации для алюминиевого сплава 7449-Т7651 (экспериментальные значения [27] обозначены кружками) по классической модели Джонсона-Кука (6) (кривая 1), критерию (1) (кривая 2) и модели Cowper-Symonds (кривая 3).

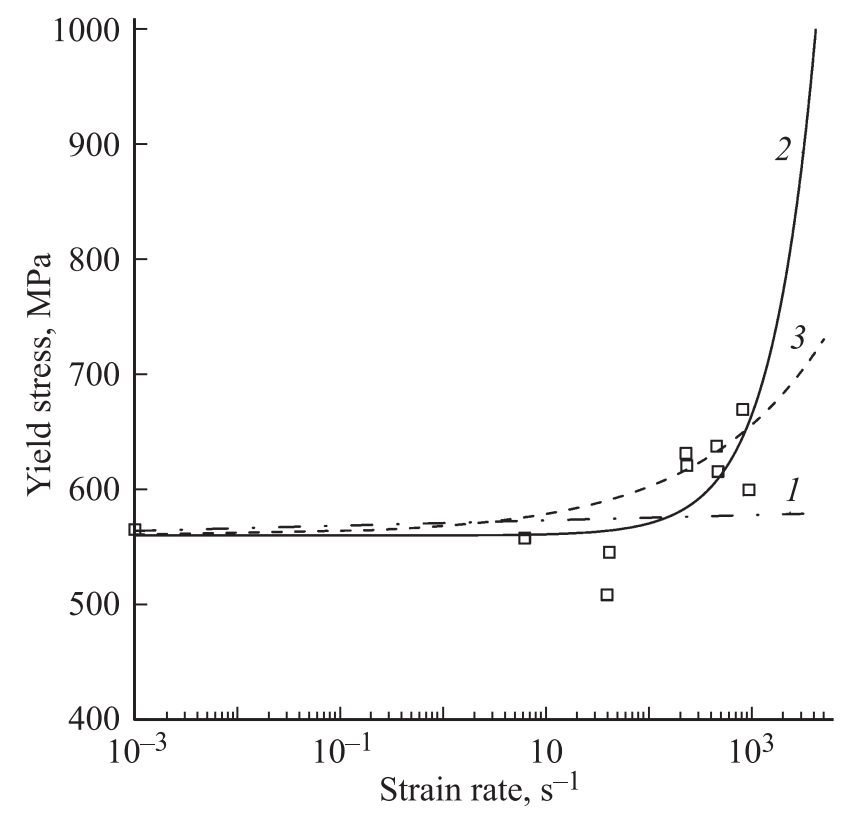

Рис. 5. Зависимость предела текучести от скорости деформации для стали В500А (экспериментальные значения [28] обозначены квадратами) по классической модели ДжонсонаКука (6) (кривая 1), критерию (1) (кривая 2) и модели Cowper-Symonds (кривая 3). 
Определение параметров модели Cowper-Symonds по характеристикам критерия (1)

\begin{tabular}{l|c|c|c|c|c}
\hline \multicolumn{1}{c|}{ Эксперимент } & $B$ & $B(\dot{\varepsilon})($ Eq. (4)) & $B$ (Eq. (5)) & q & $q($ Eq. (4)) \\
\hline Mylonas and Labeas, 2014 & 15007 & $24890\left(10^{4} \mathrm{~s}^{-1}\right)$ & 3944 & 0.95 & 2 \\
Caboni and Forni, 2015 & 141917 & $284400\left(10^{2} \mathrm{~s}^{-1}\right)$ & 1333 & 2.896 & 2
\end{tabular}

ли Джонсона-Кука (6) $\left(A_{J K}=564 \mathrm{MPa}, C=0.001769\right)$ (рис. 5).

На рис. 2 и 3 критерий инкубационного времени текучести (1) и модель Cowper-Symonds дают хорошее соответствие в определении предела текучести начиная со скорости деформации $10^{2} \mathrm{~s}^{-1}$ (рис. 2) и $10^{3} \mathrm{~s}^{-1}$ (рис. 3). В таблице показано, что оценки параметров модели Cowper-Symonds по статической ветви критерия инкубационного времени (4), где параметры зависят от скорости деформации, дают лучшее соответствие, относительно динамической ветви инкубационного времени (5).

В представленных эмпирических моделях два параметра, также как и в критерии инкубационного времени текучести. Но один из этих эмпирических параметров связывается только с параметром амплитудной чувствительности материала $(\alpha)$, а другой - с двумя параметрами критерия инкубационного времени $(\alpha, \tau)$. При этом второй эмпирический параметр связан с некоторой скоростью деформации. Таким образом, можно заметить три эмпирические константы в модели Джонсона-Кука и Cowper-Symonds. Представленные результаты в таблице показывают, что в модели Cowper-Symonds изначально две константы, но чтобы получить их по параметрам критерия можно сопоставлять их не с динамической ветвью, а со статической.

\section{4. Заключение}

Инкубационное время рассматривается как основная характеристика материала структурно-временного подхода, который предсказывает поведение предела текучести в широком диапазоне скоростей деформации.

Модель Cowper-Symonds может давать результаты, совпадающие с результатами критерия инкубационного времени текучести при определении предела текучести в зависимости от скорости деформации. Однако критерий (1) является предпочтительнее, так как параметры характеризуют два независимых друг от друга процесса: скоростную и амлитудную чувствительности материала, в отличие от параметров модели Cowper-Symonds.

Преимуществом расчетов предела текучести по критерию инкубационного времени является необходимое количество параметров, которые не требуют дальнейших модификаций на высоких скоростях деформации в отличие от эмпирических моделей Джонсона-Кука и Cowper-Symonds. Таким образом, представленный критерий инкубационного времени текучести обладает наиболее удобным численным алгоритмом предела текуче- сти в начальный момент пластической деформации, проявляя хорошее совпадение с экспериментом как в диапазоне скоростей деформаций, где реализуется эмпирическая модифицированная модель Джонсона-Кука (6), так и в существенно более широком диапазоне скоростей.

\section{Список литературы}

[1] N. Selyutina, E.N. Borodin, Yu. Petrov, A.E. Mayer. Int. J. Plast. 82, 97 (2016).

[2] G.R. Cowper, P.S. Symonds. Strain-hardening and strain rate effects in the impact loading of cantilever beams. Division of applied mathematics report. Brown University. N 28 (1957). $46 \mathrm{p}$.

[3] G.R. Johnson, W.H. Cook. In: Proceedings of the $7^{\text {th }}$ International Symposium on Ballistics. The Hague, the Netherlands (1983). P. 541.

[4] G.R. Johnson, W.H. Cook. Eng. Fract. Mech. 21, 1, 31 (1985).

[5] F.J. Zerilli, R.W. Armstrong. J. Appl. Phys. 61, 1816 (1987).

[6] D.J. Steinberg, S.G. Cochran, M.W. Guinan. J. Appl. Phys. 51, 3, 1498 (1980).

[7] D.L. Preston, D. L. Tonks, D.C. Wallance. J. Appl. Phys. 93, 211 (2003).

[8] H. Couque, R. Boulanger, F. Bornet. J. Phys. 134, 87 (2006).

[9] H. Huh, W.J. Kang. Int. J. Vehicle Design. 30, 1/2, 1 (2002).

[10] B.J. Tuathon, K.O. Bae, S.H. Lee, H.S. Shin. J. Mech. Sci. Technol. 28, 9, 3561 (2014).

[11] Z. El-Qoubaa, R. Othman. Mater. Design. 66, 336 (2015).

[12] N.S. Selyutina, Yu.V. Petrov. Dokl. Phys. 62, 2, 102 (2017).

[13] A.A. Gruzdkov, Yu.V. Petrov. Dokl. Phys. 44, 2, 114 (1999).

[14] A.A. Gruzdkov, Yu.V. Petrov, V.I. Smirnov. Phys. Solid State 44, 11, 2080 (2002).

[15] Yu.V. Petrov, Y.V. Sitnikova. Tech. Phys. 50, 8, 1034 (2005).

[16] A.A. Gruzdkov, E.V. Sitnikova, N.F. Morozov, Yu.V. Petrov. Math. Mech. Solids 14, 1/2, 72 (2009).

[17] Yu.V. Petrov, E.N. Borodin. Phys. Solid State 57, 2, 353 (2015).

[18] I.N. Borodin, Yu.V. Petrov. Mech. Solids 49, 6, 635 (2014).

[19] Yu.V. Petrov. Mech. Solids 42, 5, 692 (2007).

[20] Yu.V. Petrov, A.A. Utkin. Sov. Mater. Sci. 25, 2, 153 (1989).

[21] V.A. Bratov, A.A. Gruzdkov, S.I. Krivosheev, Yu.V. Petrov. Dokl. Phys. 49, 5, 338 (2004).

[22] A.N. Berezkin, S.I. Krivosheev, Yu.V. Petrov, A.A. Utkin. Dokl. Phys. 45, 11, 617 (2000).

[23] Yu.V. Petrov, A.A. Gruzdkov, E.V. Sitnikova. Dokl. Phys. 52, 12, 691 (2007).

[24] L. Schwer. Optional strain-rate forms for the Johnson-Cook constitutive model and the role of the parameter epsilon. $6^{\text {th }}$ Eur. LS-DYNA Users' Conf. (2007).

[25] D.N. Zhang, Q.Q. Shangguan, C.J. Xie, F. Liu. J. Alloys Comp. 619, 186 (2015).

[26] H. Couque. Phil. Trans. R. Soc. A 372, 20130218 (2014).

[27] G.I. Mylonas, G.N. Labeas. Exp. Techniques 38, 2, 26 (2014).

[28] E. Cadoni, D. Forni. EPJ Web Conf. 94, 01004 (2015). 